\title{
Evaluation and Impact Assessment of Application of Lean Six Sigma in Healthcare Service
}

\author{
Meeta M Ruparel
}

\begin{abstract}
Purpose: In the current competitive scenario, the demanding corporate strategies and the ever-rising branding world many a healthcare set-ups strive to implement the best and stand out as a benchmark. There have been many attempts to improve quality in healthcare, but most have been unsustainable. Internationally many healthcare organizations have already benefited from lean six sigma (LSS) strategies. The research aimed to deploy LSS methodologies in hospital systems to evaluate and assess the healthcare service delivery process performance on important performance criteria; namely efficiency, patient safety, care pathway, productivity, standard compliances, patient satisfaction, and employee satisfaction and further evaluate their retrospective cost Impact.
\end{abstract}

Methodology: Observation/case study (CS) techniques were used on site of stratified segment sample identified from exploratory research of only hospitals/units where LSS was allowed to be implemented; to study and analyze LSS deployment project results. A focus group feedback Pre and Post project deployment was also taken from process owners

Findings: It was statistically evident that the application of LSS methodologies significantly improves the hospital/healthcare service delivery process performance. It was observed that in an LSS deployed process project, with an improvement even in one measurable indicator that was critical to the quality of the process performance resulted in improvement of a mix of Impact Factors, which in turn resulted in its retrospective cost Impact

Originality value: The primary research was structured around the study of efficient utilization of the key resources like care time, physicians' time, manpower, material, space and equipment and management arrangements with practical project deployments of LSS methodologies. The study further addressed the problem of high incidences of adverse and sentinel events and its reductions with appropriate LSS applications in real situations.

Keywords: Green hospitals, Lean six sigma in healthcare/hospitals, Mistake-proofing in hospitals/healthcare, Patient safety in healthcare, Performance excellence in hospital services, Quality management in hospitals.

How to cite this article: Ruparel MM. Evaluation and Impact Assessment of Application of Lean Six Sigma in

\section{Research Student}

Prin LN Welingkar Institute of Management Development and Research, Mumbai, Maharashtra, India

Corresponding Author: Meeta M Ruparel, Research Student, Prin LN Welingkar Institute of Management Development and Research, Mumbai, Maharashtra, India, e-mail: meetaruparel@ rediffmail.com
Healthcare Service. Int J Res Foundation Hosp Healthc Adm 2018;6(2):74-79.

\section{Source of support: Nil}

Conflict of interest: None

\section{INTRODUCTION}

In a hospital, everything is built on care, trust, and integrity. Healthcare systems are continually innovating in clinical practices and management; however significant and sustained changes are necessary to avert the crises in healthcare quality. To meet all these demands and more concepts of branding, image building and the desire to provide quality services with cost containment and higher satisfaction for all stakeholders, more and more hospitals are on the go to implement quality initiatives to constantly improve. This could be implemented with lean six sigma (further referred to as LSS) practices in the organizations. LSS organizations have the ability and willingness to adopt contrary objectives, like reducing medical errors and getting things done faster. It is customer focused and raises performance at a breakthrough level. The benefits are evidential with quantifiable indicators of operations and profits. LSS is a complement to other quality initiatives such as ISO, JCI, $\mathrm{NABH}$, etc., and works as a tool to achieve and sustain standards requirements. ${ }^{1}$

\section{Concepts}

Six sigma's approach to quality is rigorous and is an exigent to perfection approach; defects are controlled to just 3.4 DPMO. Lean is a systematic approach to identifying and eliminating waste through continuous improvement. Lean principles and six sigma techniques work together and build on each other. LSS is the combination of improvement methods that allow organizations to achieve improvement in service, quality, cost, and profitability. Controlling healthcare cost increases, improving quality, and providing better healthcare are some of the benefits of this approach. A combination of both provides a structured improvement approach and effective tools to solve problems. This creates rapid transformational improvement at a lower cost. LSS alone may not be the 
savior of healthcare delivery, but its judicious application along clinical paths, combined with the best treatment, technology, and expertise available, will certainly improve care for the patient. ${ }^{2}$

\section{Literature Review}

Patient safety was defined by the IOM as "the prevention of harm to patients". ${ }^{3}$ Many studies confirm that medical error is prevalent in our health system and that the costs are substantial. Bates et al. found that adverse drug events were common and that serious adverse drug events were often preventable. ${ }^{4}$ A paper reviewing the safety initiatives in the health systems of the UK, Canada, Australia, and the US, indicated that these countries have all engaged in safety initiatives such as patient safety agencies, adverse event reporting and learning systems, and the use of safety performance indicators. ${ }^{5} \mathrm{~A}$ national survey of six sigma applications in US Hospitals conducted by Qianmei (May) Feng and Chris M Manuel indicated that cost benefits and improvements, typical implementation durations, efficiency and assessments related to major barriers in implementation were common LSS projects implemented in the hospitals. ${ }^{6}$ UMCG in the Netherlands made LSS an integral part of the hospital's culture and showed how an evidence-based approach to reviewing and improving procedures not only resulted in reduced costs, efficient use of resources, but also improved patient experiences. ${ }^{7}$

\section{Significance}

The rising reports of medical errors and functioning of the public and private health sector brought out the fact that there was an urgent need to prescribe and improve systems of healthcare service. Healthcare delivery deals with nothing less than the precarious balance between life and death; therefore, becomes exigent to consider exacting approaches that can significantly reduce potential errors and improve the care process. Efforts like TQM, QMS, and process re-engineering although made a noticeable impact on the healthcare environment, it, however, failed to translate into sustainable results. Currently, several LSS strategies have been endorsed as transformational by healthcare leaders and from their practices, it is evident that these efforts produce results. Such research and communication are critical to demonstrate the effectiveness and to provide insights for ensuring proper implementation in the system.

\section{METHODOLOGY}

\section{Aim}

The aim of the research was to study the application of LSS driven hospital service process and scope of clinical care pathways success by adapting LSS methodologies and tools to improve and ensure optimal healthcare quality and the highest level of patient safety in the healthcare service delivery process:

- Scope: To deploy LSS projects in real situations and study results of case studies (further referred as CS) on the application of LSS methodologies in a healthcare service delivery process and observe process performance improvements (further referred as PI) if any on applying the LSS tools and methodologies. The PI could be an overall process PI that enabled improvement in a mix of impact factors like care pathway, patient safety, standard compliance, productivity, efficiency, employee satisfaction, and patient satisfaction. The indicators measured varied from process to process with unique process specific criteria. It was observed that the process improvement resulted in performance improvement of varied impact factors, further resulting in financial gains in terms of revenue gain, indirect gain, savings gain. The study aimed to observe and analyze overall PI in a process where LSS was deployed further to observe its influences on performance impact factors

\section{Primary Research}

Observation/case study techniques were utilized and LSS projects were implemented in hospitals/healthcare units' service processes, in real situations; in the identified hospital/healthcare service unit to further study and analyze the project case results and its impact on the overall performance and its cost impact.

\section{Case Studies}

A total of 22 LSS deployment projects were deployed, on visits to sites and discussions with the hospital management of the identified hospitals.

\section{Process Owner's Insight}

A focus group feedback from process owners was taken before project start to estimate the overall performance improvement (PI) \% expected by the process owners, post LSS deployment in the applied process retrospectively; in terms of:

- "Expected PI\% (Least)": That is the minimum improvement expected

- "Expected PI \% (Desired)": That may be a stretched goal but an improvement desired.

The feedback from the process owners was also taken post- project completion to identify the performance impact factors that were influenced by the overall 
improvement, namely standard compliance, patient safety, patient satisfaction, efficiency, employee satisfaction, productivity and care pathway and its retrospective cost impact whether revenue gain, indirect gain or savings gain.

\section{Primary Research Data Analysis and Results, Interpretations}

Data of 22 case studies (CS) results of LSS deployment done during Primary research were processed and analyzed. An overview of this data analysis is discussed here.

The project results data indicated that the mean of expected PI \% (Least) was $9.42 \%$ and mean of expected PI \% (desired) was $29.27 \%$.

Graph 1 shows case study wise achieved PI \%, expected PI \% (least) and expected PI \% (desired):

- The PI \% varied from case to case from $12.09 \%$ to $100 \%$; depending on the process selected and its specific indicator measured for improvement.

- The feedback from process owners of each case revealed that:

- In 21 cases the achieved PI \% was higher than both the expected PI \% (least) and expected PI \% (desired)

- In one case (CS12) the achieved PI \% was more than expected PI \% (Least) but less than the expected PI \% (Desired).

Graph 2 is the normal probability plot of the case wise achieved PI \%. As indicated; at 95\% confidence interval $\alpha$ : 0.05 , the $p$ value: 0.249 was more than $\alpha$-level, hence statistically evident that the data followed a normal distribution. Since the achieved PI \% data fitted the normality test and as indicated followed a normal distribution; the hypothesis test was run with the one-sample T-test. The test mean required for the one-sample T-test was derived from the feedback taken by the process owners. This feedback was taken from the process owners before the project started. The mean of responses for:

- Mean expected PI \% (least) was $9.42 \%$; approximated to $10 \%$

- Mean expect PI \% (desired) was 29.27\%; approximated to $30 \%$

On brainstorming with the process owners revealed that:

- The expected PI \% (least) was the minimum improvement expected failing to meet this expectation would result in a dissatisfaction scenario

- Any improvement above that could be considered as a "satisfaction" status

An achieved improvement that is more than the expected PI \% (desired) would be a "delighted" situation and termed commendable to celebrate the success of the project.

The derived mean of expected PI \% (least) was 10\% and that of expected PI \% (desired) was 30\%. Hence the mean expected PI \% (desired) of 30\% was considered as the test mean for the hypothesis.

As derived; the hypothesis test mean; $\mu=30 \%$

\section{Hypothesis Statement}

- Ho: Application of LSS tools and methodologies has no significant impact on hospital/healthcare service delivery process performance

- Ha: Application of LSS tools and methodologies in a hospital/healthcare service delivery process results in a mean of Achieved PI \% greater than the mean of expected PI \% (desired): $30 \%$

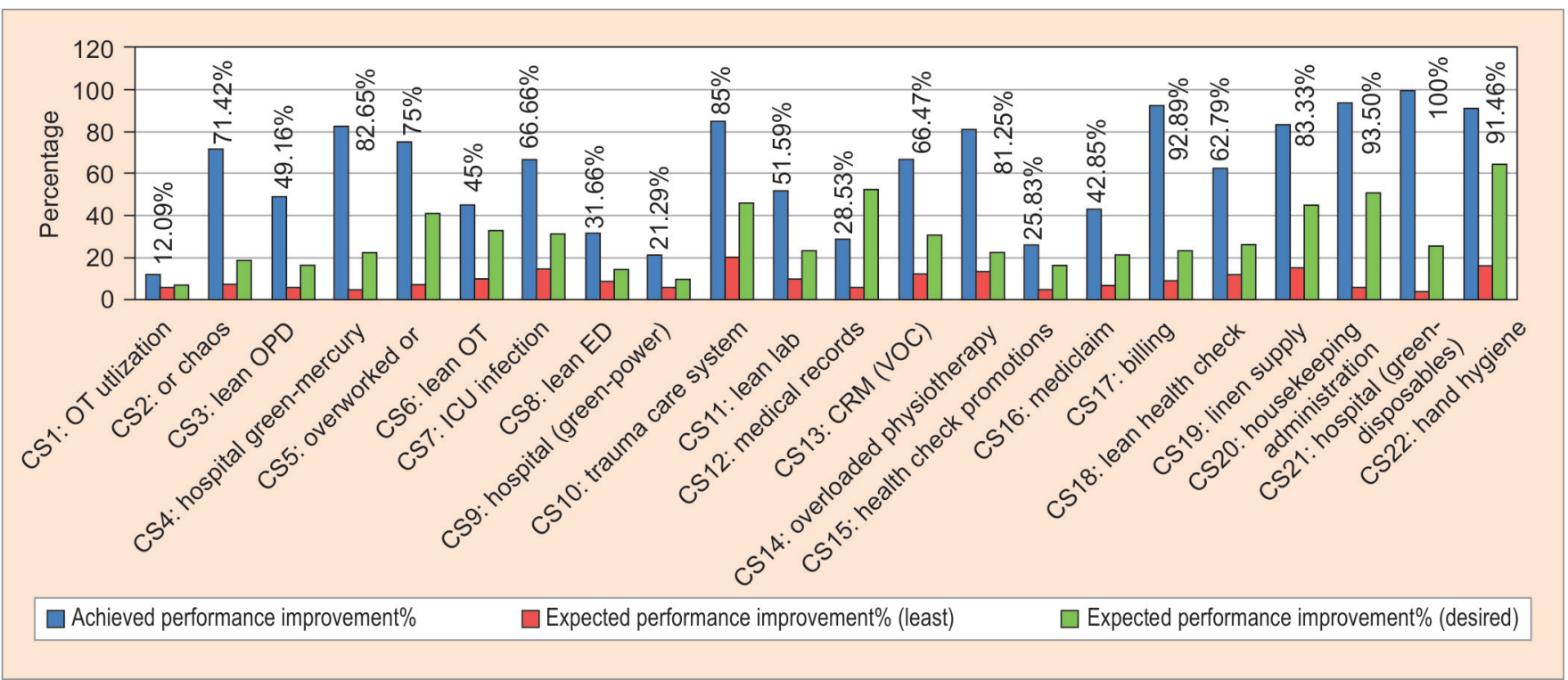

Source: Compiled by the researcher on the basis of primary data

Graph 1: Primary case study wise PI \%: achieved PI \%, expected PI \% (Least) and expected PI \% (Desired) 


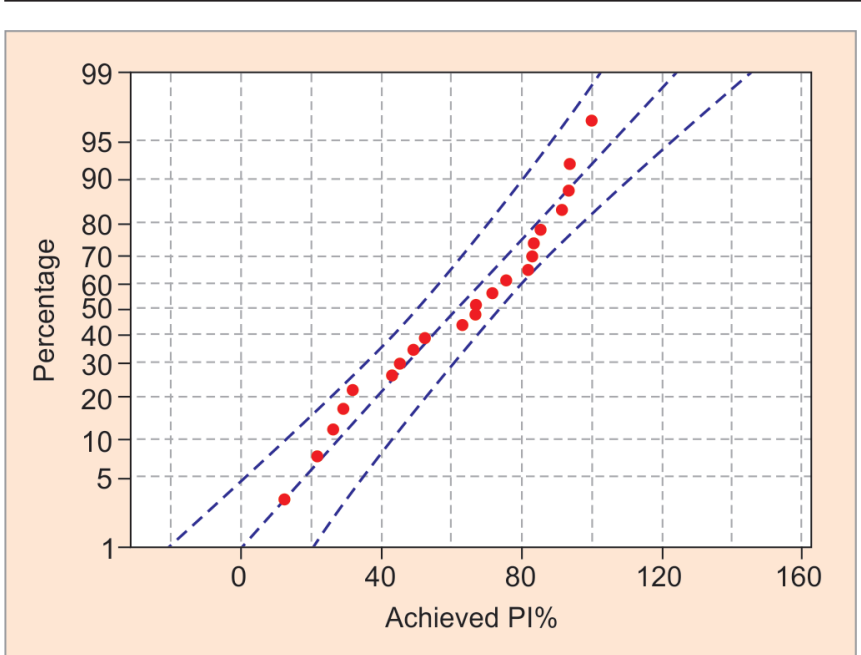

Source: Compiled by the researcher on the basis of primary data

Graph 2: Normal probability plot of the achieved PI \%

- Significance Level: Confidence level of $95.0 \%$ and test mean: 30; was considered.

\section{Hypothesis Test}

One-Sample t-test was run to test the hypothesis and determine whether Application of Lean Six Sigma tools and methodologies in a Healthcare Service Delivery process improved the process performance, i.e. to determine whether the mean of the achieved performance improvement (Achieved PI \%) was more than the defined PI \% test mean of $30 \%$.

Thus for one-sample t-test for the hypothesis test: Ho: $\mu=30$ and Ha: $\mu>30$

\section{Test results}

Table 1 is the one-sample t-test results of Achieved PI \%. The results indicated that: At a $95 \%$ confidence interval, P: $0.00001<\alpha: 0.05$. Hence the null hypothesis $\left(\mathrm{H}_{0}: \mu=30\right)$ was rejected and the alternative hypothesis $\left(\mathrm{H}_{\mathrm{a}}: \mu>30\right)$, that the mean achieved PI \% was more than the mean expected PI \% (desired): 30\%, was accepted.

A retrospective power calculation was done to analyse the statistical power of the hypothesis test. Table 2 is the retrospective statistical power calculation.

Table 1: One-sample T-test results

\begin{tabular}{|c|c|c|c|c|}
\hline \multicolumn{5}{|c|}{ One-sample T: achieved PI \% } \\
\hline \multicolumn{5}{|c|}{ Descriptive statistics } \\
\hline$N$ & Mean & St dev & SE mean & 95\% lower bound for $\mu$ \\
\hline 22 & 61.85 & 26.51 & 5.65 & 52.13 \\
\hline \multicolumn{5}{|c|}{$\mu$ : mean of achieved PI \% } \\
\hline \multicolumn{5}{|c|}{ Test } \\
\hline \multicolumn{3}{|c|}{ Null hypothesis } & \multicolumn{2}{|c|}{$\mathrm{H}_{0}: \mu=30$} \\
\hline \multicolumn{3}{|c|}{ Alternative hypothesis } & \multicolumn{2}{|c|}{$\mathrm{H}_{1}: \mu>30$} \\
\hline
\end{tabular}

\begin{tabular}{ll}
\hline T Value & $p$ value \\
\hline 5.64 & 0.00001
\end{tabular}

As indicated in Table 2, the statistical power was 0.999927. i.e., the probability of correctly rejecting the false null hypothesis was 0.999927.

As shown in Graph 3, for sample size 20, 22 and 30; considering the retrospective study test, the difference between true mean and the test mean was 31.85. For this difference: - For sample size 20, the statistical power value: 0.999789

- For sample size 22, the statistical power value: 0.999927

- For sample size 30, the statistical power value: 1.00

- Thus evident that the considered sample size of 22; was adequate for the test.

The test indicated that the true mean was greater than the test mean 52.125 at a confidence interval of $95 \%$ and Table 2 and Graph 3 indicated that there was 99.99\% chance of the significant difference of true mean and test mean, being detected with sample size: 22 .

Thus it was statistically evident that "Application of LSS tools and methodologies significantly improves the hospital/healthcare service delivery process performance".

The Dotplot in Graph 4 indicated case study wise impact factors improved and their respective cost impact, each PI resulted in improvement of a mix of impact factors that resulted in its retrospective cost impact:

- 1 Case (CS10), resulted in an improvement of a mix of six impact factors

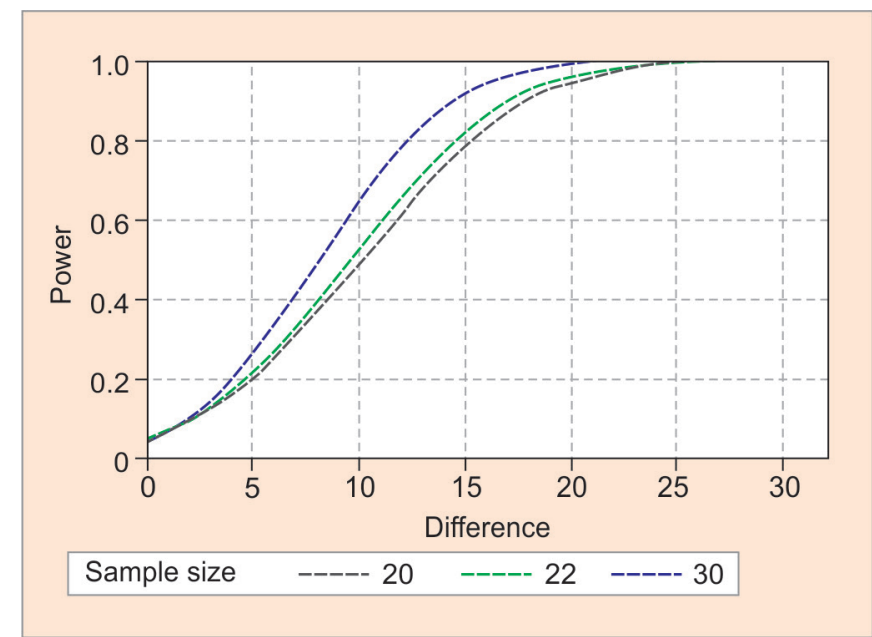

Source: Compiled by the researcher on the basis of primary data

Graph 3: Retrospective power curve for different sample size

Table 2: Retrospective power calculation

\begin{tabular}{|c|c|c|}
\hline \multicolumn{3}{|c|}{ Power and sample size } \\
\hline \multicolumn{3}{|c|}{ 1-Sample $\mathrm{t}$ test } \\
\hline \multicolumn{3}{|c|}{ Testing mean $=$ null $($ versus $>$ null) } \\
\hline \multicolumn{3}{|c|}{ Calculating power for mean $=$ null + difference } \\
\hline \multicolumn{3}{|c|}{$\alpha=0.05$ Assumed standard deviation $=26.51$} \\
\hline \multicolumn{3}{|l|}{ Results } \\
\hline Difference & Sample size & Power \\
\hline 31.85 & 22 & 0.999927 \\
\hline
\end{tabular}




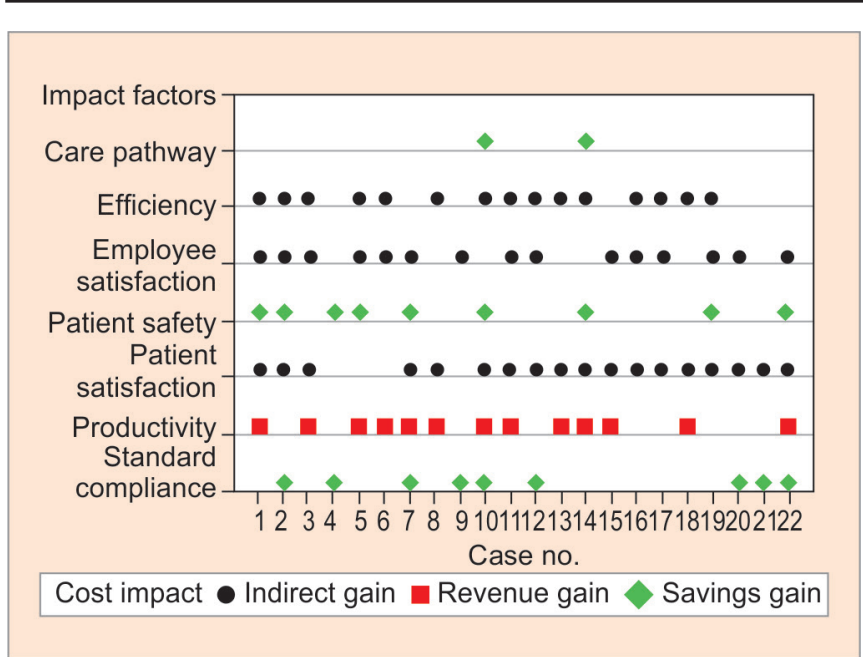

Source: Compiled by the researcher on the basis of primary data

Graph 4: Dotplot of primary case study wise impact factors and its cost impact

- Five Cases (CS1, CS2, CS7, CS14, CS22), resulted in an improvement of a mix of five impact factors

- Six Cases (CS3, CS5, CS11, CS12, CS13, CS19), resulted in an improvement of a mix of four impact factors

- Seven Cases (CS6, CS8, CS15, CS16, CS17, CS18, CS20), resulted in an improvement of a mix of three impact factors

- Three Cases (CS4, CS9, CS21), resulted in an improvement of a mix of two impact factors

The PI resulted in its retrospective cost impact in a varied manner:

- Improvement in productivity resulted in a revenue gain.

- Improvement in efficiency, employee satisfaction and patient satisfaction resulted in indirect gain.

- Improvement in patient safety, standard compliance and care pathway resulted in savings gain.

Overall improvement in 22 processes resulted in 82 improved impact factors of which 49 improved factors retrospectively resulted in indirect gain, 13 improved factors resulted in revenue gain and 20 improved factors resulted in savings gain.

\section{Observations and Findings}

- It was statistically evident that the application of LSS methodologies significantly improves the hospital/ healthcare service delivery process performance.

- LSS methodologies when applied to a healthcare service delivery process a performance improvement ranging from 12 to $100 \%$ was achieved, depending on the process, its enablers and measurable objectives.

- There was zero cases with Nil PI \%, which made it evidential that the application of LSS methodologies improved a healthcare process, although at varied PI levels.
- It was observed that the improvement varied depending on the process and its impact factors. These impact factors contributed to healthcare service performance. The study indicated that the process Improvement resulted in improving one or a mix of Impact factors namely; care pathway, standard compliance, patient safety, efficiency, productivity, patient satisfaction, and employee satisfaction and its retrospective cost impact also varied with respect to the Impact factor improved.

- It was found to be best appropriate practice to identify relevant indicators that were based on the critical to quality and process requirements, internal environment, hospital system, applicable policies, regulations, clinical case and process specific.

\section{CONCLUSION}

The research study indicated that application of LSS in healthcare improved healthcare service delivery in various ways viz., Improved care pathways, utilization of resources, increased patient safety, compliances, satisfaction scores, reduced medical errors and improved costs. LSS can be strategically deployed in various departments of a healthcare service. The processes generally focused were on the administrative systems; with creative and innovative adaptations it can impact clinical care pathways tremendously by reducing risks and opportunities of medical errors.

Any level of improvement impacted cost gains (direct/indirect).

Considering the efficacy of LSS initiative in hospitals, it was observed that LSS techniques improve the performance of most processes substantially. Even if some processes are unable to achieve expected ambitious goals, it does not diminish its importance. LSS methodologies assist in minimizing errors, achieving set quality benchmarks, optimal utilization of resources, elimination of wastes and guides as to how a set goal is achieved in terms of tangible results. Cost implications to implement LSS methodologies are very minimal compared to the losses/wastes occurring in any process variations, without any quality initiative.

\section{Future Scope}

- More application in the care delivery / clinical care process will assure optimal care process and minimum medical errors.

- More project deployment focusing specifically on particular process enabler can help identify key influencing indicators.

\section{ACKNOWLEDGMENTS}

Author takes this opportunity to thank Professor Dr PS Rao, Dean Research Department of "Prin LN Welingkar Institute of Management Development and Research, 
Mumbai (We School)"; for his patience, insightful comments and encouragements at all time of this research work. The Author is also grateful to the research department at We School for their motivating support to her at every stage of this scholarly paper preparation. The Author further extends her sincere gratitude to all the hospitals' senior management for allowing her to conduct research and accomplish real-world process improvement projects at their facilities. The Author thanks all the hospital process owners, other hospital team members and LSS green belt/yellow belt trainees for their enthusiasm and active participation during the LSS orientation training and project deployments at their respective hospitals.

\section{REFERENCES}

1. Meeta R. Six Sigma in Healthcare-An Insight to Essential Facts Hinduja Hospital Seminar conference Journal. 2008.
2. Meeta R. Lean Six Sigma in Hospitals; EHM. 2012 Aug.

3. Mitchell PH. Defining patient safety and quality care. InPatient safety and quality: An evidence-based handbook for nurses 2008 Apr. Agency for Healthcare Research and Quality (US).

4. Bates DW, Cullen DJ, Laird N, Petersen LA, Small SD, Servi $\mathrm{D}$, et al. Incidence of adverse drug events and potential adverse drug events: implications for prevention. Jama. 1995 Jul 5;274(1):29-34.

5. Arah OA, Klazinga NS, Delnoij DM, Asbroek AT, Custers T. Conceptual frameworks for health systems performance: a quest for effectiveness, quality, and improvement. International journal for quality in healthcare. 2003 Oct 1;15(5):377-398.

6. Feng $Q$, Manuel CM. Under the knife: a national survey of six sigma programs in US healthcare organizations. International Journal of Healthcare Quality Assurance. 2008 Sep 5;21(6):535-547.

7. Making it better. Strategic Direction [Internet]. Emerald; 2014 Sep 2;30(10):5-8. 